\title{
Vendre du luxe au rabais :
}

Une étude de cas dans l'hôtellerie haut de gamme à Paris

Selling discounted luxury services: a case study in a Parisian upmarket hotel

\section{Gabriele Pinna}

\section{OpenEdition}

\section{Journals}

Édition électronique

URL : https://journals.openedition.org/travailemploi/6122

DOI : 10.4000/travailemploi.6122

ISSN : 1775-416X

Éditeur

DARES - Ministère du Travail

Édition imprimée

Date de publication : 14 décembre 2013

Pagination : 21-34

ISSN : 0224-4365

Référence électronique

Gabriele Pinna, «Vendre du luxe au rabais : », Travail et Emploi [En ligne], 136 | octobre-décembre 2013, mis en ligne le 01 octobre 2015, consulté le 29 juillet 2021. URL : http://journals.openedition.org/ travailemploi/6122; DOI : https://doi.org/10.4000/travailemploi.6122 


\title{
Vendre du luxe au rabais : une étude de cas dans l'hôtellerie haut de gamme à Paris
}

\author{
Gabriele Pinna $\left.{ }^{*}\right)$
}

Cet article s'intéresse à un secteur d'activité en expansion: l'hôtellerie haut de gamme à Paris. Comme dans les entreprises situées au milieu et en bas de la hiérarchie du secteur hôtellerie-cafésrestauration, l'emploi y est souvent précaire et les salariés peu qualifiés; la formation se fait "sur le tas» et dans l'urgence; la division du travail est sexuée, ethnique, et suit la frontière entre front et back office. L'organisation ne laisse pas vraiment d'autonomie aux salariés et ne valorise pas leur professionnalisme. L'observation empirique montre que les tensions, très fréquentes, avec les clients mécontents de la qualité des services sont à mettre au compte de l'insuffisance des moyens humains et matériels engagés par l'établissement. Dans le même temps, pourtant, ces hôtels revendiquent des prestations extrêmement soignées et personnalisées, à l'image de celles offertes dans les palaces occupant le sommet du marché. C'est pourquoi la déférence et la serviabilité des salariés sont particulièrement importantes : elles permettent de sauver les apparences tout en sacralisant le «moi» des clients. L'expansion de l'hôtellerie de luxe ne s'accompagne pas d'une amélioration des conditions de travail et d'emploi mais de l'émergence d'un marché de service de luxe au rabais, reposant principalement sur l'engagement de façade des salariés.

Au cours des vingt dernières années, à Paris, le secteur de l'hôtellerie de luxe et haut de gamme a connu une croissance spectaculaire ${ }^{(1)}$. Ce phénomène est particulièrement intéressant à étudier parce qu'il est révélateur, plus généralement, d'une évolution du monde économique et des comportements de consommation des biens de luxe. L'expansion de ce secteur d'activité résulte, d'une part, de l'essor d'une nouvelle clientèle aisée en provenance des pays émergents - par exemple la Chine, la Russie, le Brésil ou l'Inde; d'autre part, elle provient d'un mouvement relatif de démocratisation du luxe, qui voit une clientèle moins fortunée accéder de plus en plus à des biens de luxe, mais seulement de façon occasionnelle ${ }^{(2)}$. Les stratèges du marketing hôtelier ne s'y sont d'ailleurs pas trompés : aux côtés de la dizaine d'hôtels détenant

(*) CRESPPA-GTM, 59-61 rue Pouchet, 75017 Paris; gabrielepinna0@gmail.com

Je voudrais remercier Léonie Hénaut dont les remarques ont été particulièrement utiles pour définir la structure de cet article.

(1) Le nombre d'hôtels de luxe a beaucoup augmenté - ils sont presque 300 en 2012 alors qu'ils étaient 158 en 2005 . De plus, le nombre des chambres a doublé en vingt ans. Les hôtels de luxe et haut de gamme représentent 18,2 \% de l'offre hôtelière parisienne. En termes de capacité d'hébergement, ils disposent de 27105 chambres, ce qui correspond à $33 \%$ des chambres de l'hôtellerie parisienne (Office du tourisme et des congrès de Paris, Le tourisme à Paris. Chiffres clés 2012). Il s'agit d'une niche de marché très importante d'un point de vue économique - ce sous-secteur réalise à lui seul plus de $60 \%$ du chiffre d'affaires du marché hôtelier parisien - mais aussi culturel et symbolique.

(2) À ce propos voir Lipovetsky, Roux (2003) et ODIT (2007). officiellement le titre de palace ${ }^{(3)}$, la catégorie des hôtels dits «haut de gamme»s'est considérablement développée. Ces hôtels pratiquent des prix moins élevés que les palaces mais revendiquent eux aussi un service de très haute qualité, soigné et sur mesure. Toutefois, le secteur de l'hôtellerie et de la restauration n'a pas connu l'accroissement de la qualification du travail qui a accompagné l'essor de nombreux secteurs d'activité pendant la période des Trente Glorieuses. Il se caractérise aujourd'hui encore par une main-d'œuvre précaire, peu expérimentée, et enregistre un taux de turnover très élevé (Monchatre, 2010). Dans ces conditions, comment l'hôtellerie haut de gamme parvient-elle à offrir un service de qualité ?

La tertiarisation de l'économie et le développement du tourisme ont favorisé un renouvellement de l'intérêt des sociologues pour le secteur «hôtellerie-cafés-restauration» (HCR). Ces quinze dernières années, le travail hôtelier a été étudié sous l'angle des rapports sociaux de genre et de la «division sexuée et ethnique du travail» (ECKERT, Monchatre, 2007; Ferreira de Macêdo, 2003;

(3) La loi du 22 juillet 2009 de « développement et de modernisation des services touristiques» - loi «Novelli» - introduit la catégorie « cinq étoiles». Puis, en 2010, l'arrêté du 8 novembre crée une «distinction Palace» alors que, traditionnellement, le titre était attribué par les professionnels du marché et par la clientèle la plus riche aux hôtels les plus prestigieux : ils se situaient au sommet du marché non seulement en vertu de l'excellence de leurs services mais aussi en raison d'une histoire et d'une renommée consolidées. 
Ferreira de Macêdo et al., 2007; Monchatre, 2006, 2010; PUECH, 2004), des qualifications et des compétences du personnel (Monchatre, 2010), de la précarité et de la santé au travail (FERREIRA DE MACÊDO et al., 2007; LADA, 2009), en lien avec la qualité et le volume de l'emploi dans une optique socio-économique (GADREY, 2002), ou encore à partir de la question de la domination dans une perspective à la fois interactionniste et néomarxiste (Sherman, 2007). Dans une démarche proche de la sociologie de Pierre Bourdieu, d'autres auteurs ont mis en évidence "l'enchantement» du rapport marchand dans le secteur touristique (GIROUD, 2007; Kotsi, 2007; Poupeau, Réau, 2007; Réau, 2006), ainsi que le processus de construction de l'offre et des pratiques touristiques à travers une analyse des trajectoires biographiques et professionnelles des fondateurs des villages de vacances (RÉAU, 2007, 2011). Malgré des différences importantes, ces recherches ont en commun d'avoir mis en évidence les conditions de travail et d'emploi particulièrement difficiles dans ce secteur, notamment pour les femmes et pour les jeunes. Le secteur de l'hôtellerie haut de gamme se différencie-t-il de l'ensemble du secteur HCR en employant un personnel stable et qualifié? Si tel n'est pas le cas, quelles sont les compétences nécessaires pour travailler, c'est-à-dire obtenir et conserver son emploi, mais aussi délivrer le service au quotidien, dans un secteur où la clientèle attend une prestation «de luxe»?

Pour répondre à ces questionnements, nous allons mobiliser les données collectées entre 2007 et 2010 dans le cadre d'un travail de doctorat sur l'hôtellerie haut de gamme à Paris. La méthode d'investigation principale a été l'observation participante : nous avons travaillé à couvert (incognito) comme salarié dans plusieurs hôtels pendant un an environ ${ }^{(4)}$. Cet article s'appuie principalement sur les données collectées dans 1'Hôtel Fashion (5) (voir encadré 1). Dans le segment haut de gamme, un nombre croissant d'hôtels cherche à moderniser et renouveler l'offre traditionnelle, notamment par le choix d'une décoration intérieure dite

(4) Deux expériences majeures de terrain ont été conduites: dans un hôtel quatre étoiles parisien, l'Hôtel Fashion, nous avons travaillé pendant six mois à temps plein en tant que night audit (voir la troisième partie de l'article pour en savoir plus sur la nature des tâches de night audit) et bagagiste-voiturier; dans un autre hôtel quatre étoiles, l'Hôtel du Prince, nous avons également occupé le poste de bagagiste-voiturier, toujours à temps plein, pendant quatre mois. Nous avons pris part à un grand nombre de conversations informelles pendant et après le travail, ce qui nous a notamment permis de collecter des données sur les origines sociales et les trajectoires professionnelles des collègues. Une vingtaine d'entretiens semi-directifs a également été réalisée avec eux. D'autres expériences plus brèves ont été menées dans des hôtels trois, quatre et cinq étoiles et dans la restauration, en qualité de bagagiste-voiturier, night audit et serveur.

(5) Les noms des hôtels et des enquêtés ont été modifiés dans un souci de confidentialité. contemporaine ou «design». Cette catégorie, dont l'Hôtel Fashion est représentatif, nous intéresse au premier chef puisqu'elle regroupe les établissements créés ces dernières années précisément pour répondre au nouvel afflux de clients évoqués plus haut, aux profils variés - vacanciers aussi bien que professionnels, occasionnels comme réguliers - mais recherchant tous un service d'un certain standing.

L'article est structuré en quatre temps. La littérature sur l'emploi et le travail dans le secteur HCR en France et dans les hôtels de luxe américains est tout d'abord analysée pour situer le modèle d'emploi propre à l'hôtellerie haut de gamme, objet de notre étude. Nous présentons ensuite le modèle organisationnel de l'Hôtel Fashion en soulignant en quoi il s'écarte de celui des palaces. En troisième lieu, nous montrons que la politique commerciale et l'inexpérience des salariés sont à l'origine d'un décalage récurrent entre le service attendu par les clients et affiché par l'hôtel, d'une part, et le service effectivement offert, d'autre part. Nous verrons, enfin, que la mobilisation des compétences relationnelles caractéristiques de la relation de service dans l'univers du luxe - déférence et serviabilité - permet aux salariés de sauver les apparences.

\section{Encadré 1 \\ Présentation de l'Hôtel Fashion}

L'Hôtel Fashion est situé dans un quartier hautement touristique de la capitale. II dispose d'une cinquantaine de chambres classées en plusieurs catégories selon la surface et l'aménagement, et notamment les caractéristiques de la salle de bain (avec douche, baignoire ou jacuzzi). Les prix varient tout au long de l'année en fonction des accords passés avec les agences de réservation sur Internet, mais aussi des offres commerciales et des promotions, ce qui génère d'importants écarts entre les prix annoncés et les prix pratiqués (inférieurs). Les prix affichés oscillent entre 200-250 euros par nuit pour une chambre classique et 1000-1100 euros pour la suite executive. Une telle politique commerciale ne constitue pas une exception. II suffit d'effectuer une recherche à l'aide d'un «comparateur de prix " sur Internet pour constater que l'Hôtel Fashion n'est pas l'hôtel haut de gamme le plus économique de la capitale. D'autres hôtels quatre étoiles offrent des tarifs bien plus avantageux. C'est le cas notamment des nombreux hôtels de chaîne qui proposent des chambres classiques à un prix fluctuant entre 100 et 150 euros. II nous semble que le marché du luxe se caractérise de plus en plus par une tension entre la recherche d'exclusivité et de distinction, d'une part - le prix élevé en est à lui seul une garantie (Veblen, 1970) - et une relative démocratisation, obtenue en augmentant le nombre des clients, attirés par des prix plus bas, d'autre part. 


\section{Les principales caractéristiques de l'emploi et du travail dans l'hôtellerie}

\section{Un emploi abondant mais peu qualifié}

Durant les trente dernières années, en France, le secteur hôtellerie-cafés-restauration (HCR) a connu une hausse considérable du nombre d'emplois (GADREY, 2002) ${ }^{(6)}$. Très prononcée dans les années 1980 , cette croissance est néanmoins plus lente dans les années 1990. Elle s'explique alors principalement par l'essor de la restauration rapide. Dans le même temps, la proportion des emplois qui y sont occupés par des salariés a augmenté suite à la diminution du nombre d'entreprises à gestion principalement familiale qui, de fait, avaient peu d'employés. Si la part des femmes au sein de la main-d'œuvre du secteur a baissé à cause de ce mouvement de salarisation de l'emploi ${ }^{(7)}$, elle demeure beaucoup plus élevée que celle enregistrée dans l'ensemble des secteurs de l'économie française (48,9\% contre $43,3 \%$ pour la période 2006-2008). Les conditions d'emploi et de travail sont néanmoins peu attractives ${ }^{(8)}$, si bien que les entreprises du secteur, même dans une période de chômage structurel, ont du mal à embaucher et, plus encore, à fidéliser leur personnel (AMINA, 2002; Molinari-Perrier, 2010).

En ce qui concerne les caractéristiques sociales du personnel, les statistiques nous fournissent des données précieuses : si la part de l'emploi féminin est plus importante que dans les autres secteurs, il en va de même pour la présence des salariés jeunes, faiblement diplômés et ayant peu d'expérience ${ }^{(9)}$.

(6) En 2010, les activités caractéristiques du tourisme emploient 857720 salariés. (Source: Rulfi D., Scherrer S. [2012], Mémento du tourisme. Édition 2011, Paris, Direction générale de la compétitivité, de l'industrie et des services.)

(7) Le taux de salarisation progresse constamment. Il est de $72,8 \%$ en 1998 , soit $12 \%$ de plus qu'en 1981 .

(8) Les salaires bruts annuels moyens pour les salariés à temps complet sont toujours parmi les plus bas en France: 22561 euros contre un salaire moyen de 31218 euros dans l'ensemble des secteurs. D'autres recherches montrent que les mauvaises conditions de travail vont de pair avec des salaires particulièrement bas (GuÉGNARD, MÉRIOT, 2009).

(9) Les actifs de moins de 30 ans sont nettement plus présents dans le secteur HCR que dans l'ensemble des autres secteurs d'activité. Parmi les salariés des cafés, hôtels et restaurants, beaucoup n'ont aucun diplôme ou ont des titres de niveau BEP (brevet d'études professionnelles), CAP (certificat d'aptitude professionnelle) ou baccalauréat, les détenteurs de diplômes de l'enseignement supérieur étant sous-représentés. En outre, $24,5 \%$ des salariés du secteur travaillent dans leur entreprise depuis moins d'un an, contre 13,5\% dans l'ensemble des secteurs. Le secteur HCR affiche un pourcentage très bas de salariés travaillant dans la même entreprise depuis plus de 10 ans : $23,5 \%$ contre $39,5 \%$ tous secteurs confondus. Enfin, le taux de rotation de la main-d'œuvre y est exceptionnellement élevé : $116,9 \%$ contre $42,4 \%$. (Source : Céreq, Portraits statistiques de branche (PSB), chiffres 2006-2008; la base PSB est accessible sur internet à l'adresse : http://www.cereq.fr/index.php/ menus/pied de page/base de donnees/Portraits-statistiquesde-branche-PSB; page consultée le 26 novembre 2013.)
En ligne avec ces éléments, Sylvie Monchatre (2010) montre, à partir de ses longues investigations dans le secteur hôtelier et dans la restauration, qu'il n'existe pas de lien étroit entre formation scolaire et trajectoires professionnelles - dans ces secteurs, la formation se fait principalement «sur le tas». Elle dévoile aussi l'existence d'un double marché de l'emploi : un marché relativement plus qualifié dont l'accès est davantage réservé aux hommes, d'une part, et un «marché transitionnel du travail» dans lequel les postes sont faiblement qualifiés, féminins, et ouverts aux salariés inexpérimentés, d'autre part. Or, la modernisation du secteur hôtelier s'accompagne de pratiques de «rationalisation industrielle» (GADREY, 1994), notamment dans l'hôtellerie de chaîne moyenne gamme, qui visent à standardiser le travail. Comme cela a été montré par MONCHATRE (2010), ce mouvement exige un moindre niveau de qualification professionnelle des salariés et limite leur capacité à accumuler une expérience valorisable sur le marché du travail. Il lisse également les différences entre emplois du front office et ceux du back office. Les tâches associées aux postes de travail sont à la fois rationalisées et simplifiées par le management, les salariés sont interchangeables et ils ne peuvent espérer une mobilité ascendante dans l'organisation dans le cadre d'une carrière professionnelle ${ }^{(10)}$. En conséquence, à mesure que le secteur HCR s'accroît, l'espace du «marché transitionnel du travail» (MonchatRe, 2010) a tendance à s'élargir.

\section{Une organisation du travail renforçant les inégalités de genre}

De nombreux travaux mettent en évidence les différences d'emploi et de conditions de travail entre les hommes et les femmes dans le secteur HCR. La qualification professionnelle et les parcours des salariés de l'hôtellerie sont en effet profondément façonnés par des assignations professionnelles sexuées qui orientent les hommes vers les emplois les plus prestigieux car considérés comme proches de l'artisanat, tel celui de cuisinier, et les femmes vers les emplois de service plus ordinaires comme celui de serveuse. Ces assignations déterminent la position du ou de la salarié(e) dans l'entreprise : «si le service est un "bien" qui s'échange sur le marché du travail, la compétence qui lui est associée s'acquiert dans le cadre des processus sociaux d'imposition, de désignation et d'affiliation qui sont au cœur de la socialisation différentielle des sexes et des classes»(Monchatre, 2010, p. 24). Les salarié(e)s issu(e)s de l'immigration en provenance d'Afrique noire, du Maghreb et d'Asie sont le plus souvent embauché(e)s en tant que femmes

(10) À ce propos, voir notre présentation à la page 25 de cet article du modèle organisationnel «hiérarchique professionnel» (SHERMAN, 2007). 
de chambre (ou bien valets ${ }^{(11)}$ ou équipiers ${ }^{(12)}$ pour les hommes) alors que les salarié(e)s d'origine française ou européenne sont régulièrement affecté(e)s au travail de front office. Le travail le plus ingrat et le plus pénible, celui qui n'est pas source de reconnaissance, est donc le plus souvent effectué par des femmes étrangères peu qualifiées. Leurs compétences d'entretien des chambres notamment ne sont pas reconnues car elles sont tenues pour "naturelles» chez ces femmes alors que d'autres tâches, également peu qualifiées mais demandant des compétences considérées comme viriles, par exemple le travail de nuit, sont remplies par des hommes, eux-mêmes issus de l'immigration (FerReira de MACÊDO, 2003).

Dans le secteur hôtelier, la gestion de l'organisation du travail renforce souvent les inégalités entre salarié(e)s (PuEch, 2004). Il n'est pas rare, en effet, que l'hôtel externalise tout ou partie du travail de ménage en faisant appel à des entreprises de soustraitance. Ces dernières peuvent même substituer au salaire horaire un système de paiement à la pièce, moins avantageux, dit «à chambre faite». D'ailleurs, comme nous l'avons constaté à l'Hôtel Fashion, les femmes de chambre sont affectées par leur entreprise à l'hôtel pour un volume d'activité donné tout en travaillant aussi, au cours de la même semaine, dans d'autres établissements. La société de sous-traitance répartit ainsi les femmes de chambre dans les différents hôtels avec lesquels elles ont des contrats en fonction des demandes des gouvernantes (13) et des taux d'occupation des chambres attendus pour le lendemain. Dans ces conditions, ballottées d'un établissement à l'autre, les salariées ont du mal à établir un lien de confiance aussi bien avec leur employeur qu'avec la direction et les autres travailleurs des hôtels dans lesquels elles interviennent. Il est fréquent que les femmes de chambre travaillent sans avoir reçu une copie de leur contrat, qu'elles reçoivent une rétribution inférieure à celle qui avait été convenue ou même qu'elles ne la reçoivent pas du tout et soient donc obligées de citer leur employeur aux prud'hommes. Les méthodes de gestion de la main-d'œuvre sont

(11) Dans l'hôtellerie, les hommes qui exercent la même fonction que les femmes de chambre sont appelés «valets».

(12) L'équipier est un salarié polyvalent qui s'occupe notamment du nettoyage des espaces communs de l'hôtel (hall, ascenseur, couloirs). Il accomplit aussi d'autres tâches selon les besoins de l'organisation, telles qu'amener des room service en chambre ou faire la plonge dans la cuisine de l'hôtel. D'après nos observations, la réalisation de ce «sale boulot» représente souvent l'enjeu de négociations et de tensions avec les bagagistes-voituriers.

(13) Les gouvernantes encadrent le travail du personnel des «étages» (femmes de chambre, équipiers, valets) et vérifient que les chambres ont été "faites» correctement avant d'en communiquer la disponibilité aux réceptionnistes. Elles peuvent aussi intervenir pour réaliser des finitions (disposer correctement un oreiller, passer un coup de chiffon sur un coin où traîne un petit peu de poussière, soigner la présentation des documents de bienvenue, etc.). expéditives et les conditions de travail suscitent les plaintes des salariées, souvent contraintes de travailler douze heures par jour, sept jours sur sept (Monchatre, 2006).

Dans ces entreprises de services, le personnel doit en outre accepter d'être très flexible. Les femmes de chambre sont souvent embauchées à temps partiel contraint - autrement dit, imposé par l'employeur -, ce qui permet aux entreprises de s'adapter plus facilement aux aléas de la demande (Puech, 2004). Henri ECKert et Sylvie Monchatre (2007) montrent que la polyvalence des femmes ne leur permet pas de sortir des "postes enclaves», c'est-à-dire des postes comportant des tâches pénibles et peu qualifiées. Par conséquent, la polyvalence ne contribue pas nécessairement à la mobilité professionnelle ascendante ni à la «mixité sociale» (ForTino, 1999). MONCHATRE (2006) s'est aussi intéressée à la relation entre polyvalence et rapport au travail en étudiant le processus de naturalisation du sur-engagement. L'implication au travail est tout simplement considérée par les employeurs comme allant de soi, ces derniers se référant à une vision des salariées comme «gardiennes du foyer» au sein de l'entreprise. La polyvalence permet à l'organisation de pallier ses défaillances, c'est-à-dire l'insuffisance des effectifs et le peu d'expérience de salariés qu'il est nécessaire de former «sur le tas» et dans l'urgence. Du point de vue des salariées, elle représente à la fois un risque, lorsqu'une tâche ingrate est à accomplir, et une opportunité d'accroître leurs compétences et d'apprendre un métier, tout en échappant à la routine.

\section{L'hôtellerie haut de gamme : la coexistence de deux modèles d'emploi}

De façon inattendue, les conditions de travail et d'emploi caractérisant globalement le secteur HCR s'observent aussi dans l'hôtel haut de gamme objet de notre étude. L'Hôtel Fashion emploie environ vingt-cinq salariés ${ }^{(14)}$, dont une quinzaine est embauchée à temps plein et en contrat à durée indéterminée (CDI). Les autres ont des statuts précaires : les femmes de chambre - d'origine indienne ou africaine, immigrées de première génération sont employées par une société de sous-traitance; l'équipier et le plongeur - des hommes issus de l'immigration asiatique, souvent du Bangladesh ou du Pakistan - ont des contrats temporaires; enfin, plusieurs étudiants en alternance renforcent l'équipe de la réception. La période d'essai est fréquemment prolongée au-delà de la durée prévue par la convention collective, les heures supplémentaires ne sont pas payées et les salariés travaillent pendant leur pause si cela s'avère nécessaire. De plus, ils ne suivent aucune formation en matière de sécurité au travail.

(14) Ce nombre peut varier selon le volume du travail prévu. 
Un tel engagement au travail ne va pas de pair avec un bon salaire ${ }^{(15)}$, ni avec de réelles possibilités de carrière. Il n'est pas étonnant alors que parmi les salariés de cet hôtel, le nombre de ceux qui possèdent une expérience de longue durée dans le secteur soit très faible. En définitive, une poignée de salariés - l'assistante de direction, la chef de réception, la gouvernante et, parfois, un ou deux réceptionnistes ou même un bagagiste - assume la gestion de l'hôtel au quotidien. Trois sont des jeunes femmes âgées de 27, 28 et 35 ans, qui ont d'ailleurs démissionné quelques années après notre enquête. Entre autres tâches, elles formaient fréquemment et dans l'urgence les nouveaux membres du personnel, et palliaient le manque d'expérience de ces nouveaux venus et l'insuffisance des effectifs en donnant de leur personne. $\mathrm{Au}$ moment de l'embauche, le reste du personnel n'avait en effet aucune expérience dans le domaine de l'hôtellerie de luxe.

Nos autres expériences de travail dans des hôtels de niveau quatre et cinq étoiles en qualité de night audit et de bagagiste-voiturier montrent que les méthodes d'embauche et de gestion de la main-d'œuvre de l'Hôtel Fashion ne sont pas exceptionnelles. Cependant, certains hôtels du segment haut de gamme offrent de meilleures conditions de travail et d'emploi, notamment en ce qu'ils procurent des possibilités de carrière à leurs salariés et recrutent du personnel plus qualifié. À l'Hôtel du Prince ${ }^{(16)}$, où nous avons enquêté à couvert pendant plusieurs mois, le personnel est beaucoup plus nombreux et expérimenté qu'à l'Hôtel Fashion. Les postes de la réception sont davantage destinés à des salariés ayant une expérience solide dans le secteur. Les candidats inexpérimentés peuvent espérer se faire embaucher en tant que bagagistes-voituriers, femmes de chambre ou équipiers en extra. L'hôtel compte entre trente-cinq et quarante salariés selon la période de la semaine ou de l'année. Néanmoins, à la différence de l'Hôtel Fashion, les salariés embauchés en $\mathrm{CDI}$ et à plein-temps sont nettement majoritaires et ne travaillent pas qu'en front office - il en va ainsi

(15) Les salariés sont payés au Smic (salaire minimum de croissance) et reçoivent, au moment de l'enquête, entre 1100 et 1300 euros nets par mois, auxquels s'ajoutent des tickets repas. L'ensemble de l'équipe de la réception partage les pourboires, qui représentent à peu près 200 euros par mois et par personne. Même les salaires des agents de maîtrise - chef de réception, responsable de la cafétéria ou gouvernante - dépassent rarement 1500 euros. Ces salariés, notamment la chef de réception et l'assistante de direction, font une dizaine d'heures supplémentaires non rémunérées par semaine, voire beaucoup plus dans les périodes où l'hôtel est complet. Il existe un écart très important entre leurs salaires et celui du manager qui gagnait, à l'époque de notre expérience dans cet hôtel, environ 4000 euros par mois.

(16) L'Hôtel du Prince dispose d'une soixantaine de chambres, classées en plusieurs catégories. Les prix des chambres classiques ne baissent jamais au-dessous des 300 euros, ce qui est supérieur aux tarifs de l'Hôtel Fashion. de quatre femmes de chambre et de trois équipiers travaillant depuis longtemps à l'Hôtel du Prince. Malgré leur ancienneté de service, ces salariés sont toutefois payés au Smic, tandis que les personnels $\mathrm{du}$ front office perçoivent des salaires plus élevés, des primes, et un intéressement sur les profits de l'hôtel (17).

Les conditions de travail et d'emploi ne sont donc pas homogènes au sein du segment haut de gamme du secteur hôtelier. Cette observation recoupe l'analyse réalisée par Rachel SHERMAN (2007) à propos des palaces américains ${ }^{(18)}$. Elle identifie en effet deux modèles d'organisation contrastés. D'un côté, les hôtels qui offrent les meilleures prestations ont, comme à l'Hôtel du Prince, une organisation du travail de type «hiérarchique professionnel». Dans ce modèle, la progression dans la carrière professionnelle - par exemple, beaucoup de salariés démarrent leur carrière en tant que groom pour devenir par la suite bagagiste ou voiturier, et enfin assistant concierge ou même chef concierge - est valorisée par la direction. À l'autre extrême, le modèle de "flexibilité informelle» est caractérisé par la porosité des frontières entre les postes de travail et les métiers. Comme à l'Hôtel Fashion, la division horizontale du travail d'accueil et d'accompagnement des clients n'est donc pas particulièrement marquée, et la polyvalence est de règle. Cependant, dans tous les palaces étudiés par SHERMAN, les inégalités entre salariés, évoquées précédemment, sont aussi en vigueur.

Enfin, il semble que l'hôtellerie, à l'exception de certains hôtels relevant du segment supérieur haut de gamme et s'efforçant d'adopter les standards propres aux établissements les plus prestigieux, s'appuie sur une organisation du travail profondément façonnée par des assignations professionnelles liées au sexe, à l'âge, à l'origine ethnique et au niveau de diplôme des salariés ${ }^{(19)}$. L'organisation du travail et la frontière rigide entre front et back office suivent

(17) De plus, les pourboires y étaient beaucoup plus élevés qu'à l'Hôtel Fashion. Certains mois, nous avons ainsi presque doublé notre salaire. Plus généralement, d'après des collègues ayant de l'ancienneté, un bagagiste-voiturier gagne en moyenne entre 600 et 700 euros par mois en pourboires.

(18) Les palaces en France n'ont pas fait jusqu'à présent l'objet d'une étude approfondie. Deux jeunes chercheurs s'intéressent actuellement, dans le cadre de leurs thèses de doctorat, à l'hôtellerie de luxe: Thibaut Menoux (CSE-EHESS) étudie la profession des concierges dont l'autonomie est remise en question par le processus de modernisation des hôtels et Amélie Beaumont (CSU-CRESPPA) s'intéresse à la socialisation politique des salariés de l'hôtellerie de luxe. Par ailleurs, les articles d'Henri Peretz $(1992,2005)$ éclairent à la fois le rapport au travail et les relations avec les clients des vendeurs et vendeuses des boutiques de luxe parisiennes, ainsi que les transformations de l'organisation du travail et de la relation de service qui interviennent pendant les périodes de soldes dans ces boutiques.

(19) La division sociale du travail à la fois ethnique et sexuée est encore plus marquée dans les localités touristiques balnéaires des pays du Sud, où la main-d'œuvre locale ou immigrée travaille au service des clients, qui ne se rendent dans ces localités que pour y passer leurs vacances (ADLER, 2004). 
ainsi une «division sexuée et ethnique du travail» très marquée, des deux côtés de l'Atlantique ${ }^{(20)}$ et toutes catégories d'hôtels confondues. Surtout, il est surprenant de voir que le «marché transitionnel du travail» (Monchatre, 2010) dans lequel les salariés sont peu qualifiés voire inexpérimentés constitue un réservoir de main-d'œuvre aussi important pour des entreprises hôtelières censées offrir un service de «haute qualité», ou tout au moins de qualité supérieure à celle du service des hôtels de moyenne gamme. Ce hiatus entre le modèle d'emploi suivi par les hôtels haut de gamme et la qualité affichée est à l'origine de nombreuses tensions et défaillances dans l'organisation de l'activité au quotidien.

\section{L'organisation du travail à l'Hôtel Fashion : injonctions contradictoires et tensions entre salariés}

À l'Hôtel Fashion, le travail est divisé en trois secteurs - réception et conciergerie, «étages», et cafétéria-bar-restaurant - correspondant aux trois fonctions majeures de l'organisation : accueillir et accompagner les clients pendant leur séjour; préparer et nettoyer les chambres et les parties communes de l'hôtel; servir de la nourriture et des boissons pendant les séjours des clients. La comptabilité est partiellement externalisée. Un technicien s'occupe, enfin, de l'entretien et de la maintenance de l'hôtel (21). Dans les hôtels qui disposent d'un nombre plus important de chambres, l'entretien constitue un secteur à part entière. Si cette organisation du travail en trois ou quatre secteurs se retrouve dans tous les hôtels de luxe, elle diffère, selon les endroits, principalement en fonction du ratio entre nombre de chambres et nombre de salariés : la division du travail est ainsi plus marquée et la spécialisation productive des salariés plus importante dans les hôtels où le ratio est davantage favorable au nombre de salariés. Dans les palaces, ce ratio prévoit au

(20) Dans les palaces étudiés par SHERMAN, les salariés du back office sont le plus souvent des femmes étrangères venant d'Asie ou d'Amérique du Sud, ou des femmes afro-américaines, alors que les salariés du front office, plus jeunes et diplômés, sont la plupart du temps blancs, américains ou européens. Les clients ne récompensent que très rarement le travail des salariés du back office par des pourboires, ce qui renforce l'invisibilité de leur travail.

(21) Précisons toutefois que, pendant plusieurs semaines, aucun technicien n'a été embauché, faute de candidats prêts à accepter les conditions de travail et d'emploi prévues par le management. De plus, à l'époque où un technicien était en poste, il ne travaillait qu'à temps partiel car il s'occupait également d'un autre hôtel, appartenant au même groupe. Les problèmes techniques de maintenance étaient pourtant nombreux. En l'absence de technicien, c'est un bagagiste débrouillard qui intervenait, à la demande de la chef de réception, afin d'effectuer des réparations urgentes - et, à bien des égards, improvisées - dans les chambres. Par exemple, lorsqu'une fuite d'eau était signalée, il essayait de la réparer en se servant de ruban adhésif, sous les yeux stupéfaits des clients. moins trois salariés pour chaque chambre. C'est pourquoi il est possible, par exemple, d'y distinguer le travail des réceptionnistes de celui des concierges. La spécialisation du travail d'accueil et d'accompagnement des clients permet de décomposer les fonctions des employés du hall en plusieurs postes de travail, chacun étant chargé de différentes fonctions, hautement symboliques, comme c'est le cas du groom, du bagagiste, du voiturier, du chausseur ou, anciennement, du liftier(22). Dans l'hôtel objet de notre étude, le ratio est inversé : au lieu de trois salariés par chambre, l'hôtel compte environ un salarié pour deux chambres, voire plus. Dans cette partie, nous allons voir que les stratégies de la direction sont à l'origine de contradictions dommageables à la réalisation du service, et de tensions entre les salariés.

\section{Personnaliser le service et répondre aux aléas dans un contexte de travail standardisé : des injonctions contradictoires}

D'après les travaux de Jean GADREY (1994) sur l'évolution des entreprises de services dans les sociétés contemporaines, le modèle dominant dans l'hôtellerie haut de gamme devrait être celui de la «rationalisation professionnelle». Confronté à des demandes très variées, à des situations imprévues et à des clients aux profils très hétérogènes, le personnel du front office devrait en effet disposer d'une certaine autonomie dans l'exercice de son travail, et s'appuyer sur un travail cognitif de typification des cas. Nous avons d'ailleurs pu constater que les concierges de l'Hôtel du Prince, comme ceux des palaces étudiés par SHERMAN (2007), répondent aux clients en s'appuyant pour ce faire sur un ensemble de catégories qui mêlent la nationalité, l'âge, les origines sociales ou encore la situation familiale. Ces catégories informelles, issues de l'expérience, sont élaborées et constamment ajustées à travers les échanges avec les clients mais aussi à la faveur d'un travail de réflexion collective avec les collègues, et non pas par la direction. Elles permettent de réduire les aléas et fournissent des repères fondamentaux aussi bien en termes de réussite professionnelle - en augmentant les chances de comprendre les exigences des clients - qu'en termes d'économie psychique des salariés. Ajoutons que, dans un contexte de «rationalisation professionnelle», les professionnels ont tendance à s'organiser sur le modèle des professions établies en définissant leurs conditions

(22) L'employé qui appuie sur le bouton d'appel au lieu des clients dans l'ascenseur ( "lift» en anglais). 
d'accès et d'exercice (GADREY, 1994), ce qui est le cas, du moins partiellement, des concierges ${ }^{(23)}$.

Cependant, dans l'hôtel objet de notre étude, la rationalisation du travail de service ne semble pas se rapprocher de ce modèle. Tout d'abord, les salariés n'y sont pas adhérents d'organisations professionnelles ou syndicales et l'accès de non-professionnels aux métiers du front office est assez fréquent, comme nous l'avons vu. Ensuite, le management essaie de planifier leur travail dans les moindres détails. La logique de l'organisation, avec ses contraintes et ses paradoxes, l'emporte largement sur l'autonomie des salariés. Le travail des salariés de la réception, en particulier, est réglé selon un modèle néotaylorien à peine adapté à l'univers des services, qui va à l'encontre de celui de la «rationalisation professionnelle» décrit par GADREY (1994). Des fiches de poste détaillent un ensemble de tâches administratives et bureaucratiques à réaliser à des horaires préétablis tout au long du service. En dehors de l'arrivée et du départ des clients (24), ces fiches ne comportent aucune référence au travail que suppose la réponse à leurs demandes ou attentes, ce qui révèle une contradiction aiguë entre logique de service et logique bureaucratique: alors que le travail du personnel est censé être entièrement orienté vers la satisfaction et l'accompagnement des clients, il est dans les faits davantage dicté par les standards dont se dote l'organisation. Les réceptionnistes et les night audit planifient ainsi leur temps de travail en fonction de la réalisation des tâches prévues par les fiches, et interrompent l'accomplissement de ces tâches administratives chaque fois qu'un client leur pose une question, à la réception ou par téléphone. Paradoxalement, le client devient une variable imprévisible empêchant le salarié d'effectuer efficacement son propre travail bureaucratico-administratif! Pour ces raisons, le temps de

(23) Le concierge accompagne les clients avant, pendant et après leur séjour et dispose d'un temps suffisant pour établir des relations personnalisées avec eux. Il se doit de bien connaître la vie sociale et culturelle parisienne afin d'être en mesure de conseiller aux clients des activités susceptibles de les satisfaire. La figure du concierge est caractérisée par sa capacité, acquise par l'expérience de travail dans l'hôtellerie, à comprendre les clients à partir d'une analyse fine de leur profil. De plus, le concierge gère toute une gamme de prestations pour les clients, qu'il s'agisse d'une demande de garde d'enfants, de la réservation d'une table pour un restaurant renommé ou même de l'achat de places pour une représentation le soir même à l'opéra. Les concierges disposent d'un capital social important grâce à leur appartenance à un vaste réseau de professionnels de la vie sociale parisienne. Ils sont très souvent membres d'une association internationale de concierges, "Les clefs d'or». L'accès à cette confrérie est assez strict : il faut constituer un solide dossier à partir des lettres de remerciement des clients et il est indispensable d'être parrainé par deux membres.

(24) Des fiches spécifiques sont remises aux salariés afin de standardiser leurs interactions avec les clients lors du checkin et du check-out. Pour ces deux moments particulièrement importants, les salariés reçoivent des indications très détaillées sur ce qu'il faut dire et faire, en respectant des séquences précises. travail du personnel du front office est continuellement morcelé.

À l'Hôtel Fashion, non seulement l'organisation ne s'appuie pas sur les professions phares de l'hôtellerie, comme celle de concierge, mais les activités moins prestigieuses, par exemple celles de réceptionniste, bagagiste ou voiturier, ne donnent pas lieu à une division du travail strictement spécialisée : les salariés sont très polyvalents. Réaliser des tâches de conciergerie pourrait contribuer à la fois à améliorer l'image qu'ont les salariés d'euxmêmes et les représentations qu'ont les clients des réceptionnistes, dont le métier, considéré comme «féminin», est dévalorisé. Mais les choses ne se passent pas vraiment ainsi. Les réceptionnistes assument un faisceau de tâches tellement diversifiées qu'ils sont constamment pris, comme nous l'avons dit plus haut, entre le travail bureaucratique - réservations, préautorisations, check-in, check-out, facturations, gestion des contrats avec les agences de réservation, archivage des dossiers, attribution des chambres, préparation des lettres de bienvenue, etc. - et les interactions avec les clients. Dans ces conditions, endosser le rôle de concierge ne contribue pas nécessairement à une plus grande reconnaissance du personnel du front office car le temps manque pour bien s'occuper du client. De plus, ce travail d'accompagnement sur mesure est fréquemment réalisé par des réceptionnistes qui ne possèdent une connaissance approfondie ni des clients ni de la vie sociale et culturelle parisienne. Il n'est pas étonnant alors que les réponses aux demandes soient standardisées : les réceptionnistes donnent les mêmes informations à n'importe quel client alors que l'enjeu devrait être, si l'on suit les préceptes du marketing, de les adapter au profil et à l'individualité de chacun d'entre eux. En outre, la gestion des salariés est assez expéditive; nous avons par ailleurs remarqué, de manière générale, que le manager et les responsables des différents secteurs ne font pas vraiment confiance à leurs subordonnés, et d'autant moins que, parmi eux, nombreux sont ceux qui ne travailleront à l'hôtel que pendant une période limitée. Les salariés sont régulièrement jugés seuls responsables des erreurs commises et du mécontentement des clients, et sont assez souvent durement réprimandés en public, que ce soit devant leurs collègues ou devant les clients.

\section{Des tensions entre les postes et les secteurs}

Par des entretiens individuels, l'analyse du cahier des plaintes des clients, des réunions « ouvertes» aux remarques du personnel, etc., le manager de l'hôtel exerce une certaine pression sur les salariés afin de les amener vers une démarche qualité. Cependant, de manière frappante, cet appel à la coopération et à la communication ne concerne que les seuls membres de la réception. Alors qu'ils participent pourtant au travail d'accueil et d'accompagnement 


\section{Encadré 2}

\section{Approvisionnement et gestion du minibar : manquements en cascade (1)}

Cet exemple est particulièrement éclairant pour illustrer la complexité du travail de service. Le minibar(2) est un service payant proposé aux clients qui sont censés le trouver rempli dès leur arrivée en chambre (à partir de 14 heures ou même avant). L'approvisionnement et la gestion du minibar impliquent la coopération entre plusieurs salariés : femmes de chambre, bagagistes-voituriers et réceptionnistes. Tout d'abord, les femmes de chambre ont pour charge de remplir des fiches récapitulant les consommations des clients. Elles doivent ensuite les déposer à l'accueil de manière à ce que les réceptionnistes facturent les consommations des clients le matin avant leur départ. Premier signe de désintérêt envers cette tâche : les femmes de chambre ne remettent pas les fiches; il leur arrive de les garder dans leurs chariots ou de les déposer tout simplement dans le monte-charge. Par ailleurs, pourquoi les femmes de chambre ne remplacent-elles pas les boissons qui ont été consommées par les clients? Parce que le manager ne leur fait pas confiance : l'accès au dépôt des boissons est interdit à la plupart des salariés, en raison des vols qui sont assez fréquents dans l'hôtellerie. Ainsi, dans l'hôtel objet d'étude, la mission incombe aux bagagistes-voituriers dont la première tâche en début d'après-midi consiste à faire un tour avec un chariot très lourd, chargé de boissons, pour réassortir les minibars. La stratégie adoptée par les bagagistes afin d'éviter de faire de nombreux allers-retours entre le chariot ${ }^{(3)}$ (rangé à côté de l'ascenseur) et les chambres, consiste à remplir un gros sac avec toutes les boissons signalées comme manquantes dans les minibars de toutes les chambres d'un même étage. De cette manière, ils peuvent ne faire qu'une seule tournée afin de se dépêcher.

Les bagagistes-voituriers accomplissent cette tâche précipitamment, si bien que les clients s'installent parfois en chambre et demandent des boissons qu'ils ne trouvent pas dans leur minibar. La chef de réception invite pourtant les bagagistes-voituriers à s'acquitter de l'approvisionnement des minibars le plus rapidement possible. Mais, avec toutes les interruptions dues à la dynamique du travail qui implique de donner la priorité aux voitures et aux bagages des clients, cette mission peut les occuper entre une et deux heures. D'autant qu'ils sont confrontés au problème suivant : lorsqu'ils vérifient les minibars, ils constatent quelquefois que les indications des femmes de chambres sur les fiches ne sont pas exactes. Les bagagistes-voituriers ont alors le choix ou bien de corriger les fiches et remplacer les boissons effectivement manquantes; ou bien de ne pas ralentir leur rythme de travail en suivant à la lettre les informations des fiches. Les bagagistes n'ont pas de stratégie unique; ils s'adaptent en fonction de leur charge de travail et de leur conscience professionnelle qui dépend de leur rapport au travail, plus ou moins positif. Cet enchaînement de manquements dans les tâches à accomplir a plusieurs conséquences : d'une part, les plaintes des clients qui n'ont pas à disposition les boissons souhaitées dans leurs minibars; d'autre part, un manque à gagner pour l'hôtel dans un domaine - celui des boissons - qui est normalement source de profits pour les entreprises du secteur HCR. Nous avons observé comment, lors du check-out, les clients déclarent régulièrement ne pas avoir effectué les consommations indiquées sur leur note. Les réceptionnistes se trouvent alors dans une position délicate : la distance sociale qui les sépare des clients les invite à la prudence; de plus, conscients que les données des fiches des minibars ne sont pas fiables, ils préfèrent annuler les consommations des clients.

(1) Nous présentons cette étude de cas parce qu'elle nous paraît constituer un très bon exemple des tensions entre front et back office. Cependant, notre démarche étant qualitative, nous n'avons nullement l'intention d'étendre les conclusions de cette analyse à l'ensemble des hôtels de luxe parisiens. Il est possible en revanche de généraliser les tensions liées à l'organisation et à la division sociale du travail, sachant que celles-ci peuvent prendre la forme de différentes controverses.

(2) Il contient quatre bouteilles d'eau, deux de jus de fruit, deux de Coca-Cola, deux bières, une bouteille de vin blanc de Bordeaux, deux demi-bouteilles de Champagne, six mini-bouteilles de boissons alcoolisées, des bonbons, des chocolats et des cacahuètes.

(3) Ils ne peuvent pas déplacer le chariot avec eux parce qu'il est très lourd et bruyant; de plus, les couloirs sont encombrés des chariots des femmes de chambre; ils ne pourraient donc pas toujours le faire passer.

des clients, les bagagistes en sont parfois exclus. Et surtout, l'ensemble du personnel du back office en est tenu à l'écart, du moins dans les hôtels étudiés. Pourtant, étant donné que la plupart des services sont réalisés grâce à la coopération entre front et back office, coordination et échange d'informations sont indispensables afin d'assurer la réactivité de l'organisation.

Les frontières rigides entre les secteurs et les postes de l'établissement, qui recoupent une «division sexuée et ethnique du travail» très marquée, sont à l'origine d'erreurs dues à une mauvaise communication et à une faible coopération horizontale entre les salariés. Dans un contexte de travail aussi difficile, les salariés, surmenés et insatisfaits, cherchent très certainement avant tout à se protéger. Les disputes, parfois violentes, autour des tâches supplémentaires et du «sale boulot» sont assez fréquentes, par exemple, entre les femmes de chambre et les équipiers, ou bien entre les équipiers et les bagagistes - notamment, à propos de qui, pendant l'après-midi, doit faire la vaisselle du room service -, ou bien encore, entre les femmes de chambre et l'agent d'entretien accusé de salir les chambres déjà nettoyées - et, en général, entre les salariés du front et du back office. Un simple verre sale à laver peut déclencher un véritable conflit dans les coulisses de l'hôtel. Il n'est pas étonnant alors que des salariés 
pourtant polyvalents décident de ne pas accomplir une tâche additionnelle qui ne donne lieu à aucune forme de reconnaissance et ne fait peser aucun risque de sanction (à la différence de celles réalisées en interaction avec les clients). Le luxe se manifestant notamment dans l'attention portée aux détails, les manquements répétés de la part des salariés (voir encadré 2) ont de fortes chances de se transformer en un service de qualité douteuse aux yeux de clients très exigeants.

\section{Logique commerciale et qualité du service}

Les tensions entre la rigidité dont font preuve l'encadrement et la hiérarchie et la souplesse indispensable pour travailler en interaction avec des clients très exigeants s'inscrivent au sein d'une stratégie commerciale destinée à obtenir des profits immédiats (voir encadré 3). Afin de satisfaire les propriétaires de l'hôtel souhaitant rentabiliser rapidement leur investissement immobilier, dans l'espoir de vendre le fonds de commerce à un prix élevé au bout de quelques années, le management réduit les coûts du travail et d'entretien de l'immeuble. Le nombre de salariés est structurellement insuffisant pour faire face, de manière efficace, aux nombreuses demandes des clients. Pendant les «coups de feu», ces derniers sont souvent obligés d'attendre : attendre devant l'hôtel qu'un bagagiste prenne en charge leurs bagages ou leur voiture, attendre à la réception que la chambre soit prête lorsque l'hôtel est complet ou attendre qu'un taxi arrive pour les emmener à destination. De plus, lorsque l'hôtel est complet et que le nombre de départs et d'arrivées est important, il y a trop peu de femmes de chambre pour nettoyer l'ensemble des chambres avant 14 heures - heure à laquelle, pourtant, le check-in est censé pouvoir commencer. Les salariés assurant la réception n'ont alors plus qu'à espérer que les clients ne se présentent pas trop tôt parce qu'ils risqueraient de ne pas trouver leur chambre prête. Par ailleurs, l'Hôtel Fashion n'a pas de convention d'abonnement avec une compagnie de taxis parisienne car "cela coûte trop cher», selon les mots du manager. Les réceptionnistes sont donc obligés d'effectuer les réservations en passant par les standards téléphoniques ordinaires des compagnies de taxis, si bien que leurs appels ne sont pas prioritaires. Les soirs de week-end, il n'est pas rare que plusieurs clients demandent un taxi en même temps; or, les lignes des compagnies étant particulièrement saturées à cette période de la semaine, l'attente avant d'obtenir un taxi peut avoisiner une demi-heure, voire davantage. Les clients s'impatientant dans le hall, la direction n'a trouvé d'autre solution que d'envoyer le bagagiste dans la rue adjacente, souvent sous la pluie, en espérant qu'il parvienne à stopper un taxi de passage.

\section{Encadré 3}

\section{Incidents de surbooking de l'Hôtel Fashion}

Une bonne partie des clients réserve les chambres sur Internet, en passant par des agences de réservation en ligne qui imposent à l'hôtel de laisser, à disposition des acheteurs potentiels, un certain nombre de chambres par jour. Cependant, comme le manager cherche à atteindre des taux d'occupation importants, lorsque le nombre de chambres disponibles est très limité, la vente n'est pas bloquée. De fait, l'hôtel est régulièrement surbooké : comme les compagnies aériennes, les hôtels disposent en effet d'un volant de chambres potentiellement libres car ils enregistrent régulièrement des no-show (des clients qui ne viendront pas, même s'ils ont réservé une chambre). Or cette marge de manœuvre n'est pas suffisante pour couvrir les risques encourus par cette politique commerciale agressive : dans les périodes chargées (week-ends, vacances, salons ou événements sportifs), certains clients, qui ont pourtant réservé et même payé leur séjour, ne pourront pas être hébergés à l'hôtel. Les réceptionnistes de l'Hôtel Fashion se retrouvent donc à devoir se débrouiller pour reloger ces clients surnuméraires. Même si l'hôtel prend en charge la recherche et la réservation d'un taxi pour les amener à leur nouvel hôtel, les clients sont déçus et mécontents d'avoir à changer de lieu, notamment lorsqu'ils ont fait de longs voyages en avion avant d'arriver.

À l'Hôtel du Prince en revanche, cette situation se présente assez rarement. En effet, l'hôtel s'appuie moins sur les agences de réservation sur Internet. Les clients sont souvent des habitués et beaucoup parmi eux appellent directement la réception de l'hôtel pour réserver une chambre. Le directeur de l'hôtel affirme aussi préférer ne pas atteindre la pleine occupation des chambres plutôt que d'être contraint de rediriger les clients vers un autre hôtel.

Quelles que soient les attentes des clients, le taux de rotation du personnel et l'embauche de salariés inexpérimentés se conjuguent pour générer des erreurs. La plupart des salariés de l'Hôtel Fashion n'y travaillent que pour des raisons strictement alimentaires. Ils ont des profils variés : étudiants, français et étrangers, en master ou en doctorat ${ }^{(25)}$; salariés titulaires d'un diplôme de l'enseignement supérieur - nous pouvons citer les cas d'un avocat espagnol et d'un ancien cadre de la finance d'origine maghrébine - qui travaillent dans l'hôtellerie tout en cherchant un autre emploi; salariés qui n'ont pas fait de longues études mais rêvent d'une carrière artistique - D.Js, peintres, musiciens, écrivains. Si leur passage dans l'hôtellerie venait à se poursuivre - tous, d'ailleurs, craignent de rester «coincés» dans ce secteur -, ils pourront gagner la confiance de leurs collègues plus

(25) Le manager préfère embaucher des étudiants «âgés», car les plus jeunes sont considérés peu fiables et souvent pas à la hauteur d'un service haut de gamme. 
expérimentés. Étant donné leurs compétences linguistiques, informatiques et relationnelles, ces salariés sont régulièrement embauchés en tant que night audit, une activité qui ressemble à celles des réceptionnistes tout en comportant aussi une part de «sale boulot» (Hughes, 1996) - par exemple, sortir les poubelles ou dresser les tables pour le petit-déjeuner. Or, le salarié occupant le poste de night audit étant tout seul de 1 heure à 7 heures du matin, il lui est impossible de recourir au soutien de ses collègues en cas de besoin, ce qui peut avoir des conséquences fâcheuses comme dans le cas suivant (voir encadré 4).

\section{Encadré 4}

\section{Le poste de night audit ou les aléas d'une trop grande solitude}

Un collègue d'origine étrangère, venu à Paris pour faire des études, occupait ce soir-là le poste de night audit. Vers 2 heures du matin, il est sorti de l'hôtel pour orienter des clients devant se rendre dans un restaurant du quartier. Ce faisant, il oublia de retirer les clés de la porte d'entrée, programmée pour se verrouiller entre minuit et 5 heures du matin. II s'est donc retrouvé bloqué à l'extérieur de l'hôtel si bien que les clients rentrés après l'incident n'ont pas eu accès à leurs chambres... Le collègue s'est vu contraint d'attendre la réouverture automatique des portes car non seulement il n'avait pas sur lui le numéro du manager de l'hôtel, mais de plus, les pompiers n'interviennent qu'en cas d'accident. II a donc pris l'initiative d'adresser les clients vers un autre hôtel en assurant que l'Hôtel Fashion couvrirait les frais de leur séjour ${ }^{(1)}$. Ce soir-là, le salarié était fautif car il n'avait pas respecté une règle très simple du métier : ne pas se séparer d'un passe général, des clés de la porte d'entrée et d'un talkie-walkie lors de chaque déplacement. Cela paraît aller de soi mais la formation au métier est très rapide, ne dure bien souvent qu'une nuit et est assurée par un salarié lui aussi inexpérimenté. Certaines règles du métier s'apprennent donc «sur le tas" et aux dépens des salariés. Or, d'après nous, ce sont justement ces compétences, qui leur permettent de faire face à d'innombrables stimuli et demandes en répondant de façon automatique et sans hésitation. Par exemple, un collègue plus expérimenté que nous, nous fera remarquer combien, en aucun cas, il ne faudrait monter dans l'ascenseur la nuit : «Tu fais quoi s'il tombe en panne?»

(1) Le lendemain, le salarié a été convoqué par le manager. Il a tout de suite annoncé son intention de payer lui-même les dommages causés. En général, les salariés sont censés payer de leur poche lorsque leurs erreurs - réelles ou supposées - provoquent des pertes à l'hôtel : par exemple, si un client demande un taxi et ensuite décide de partir sans l'attendre, le salarié peut être appelé à payer lui-même cette course.

Le management doit donc faire preuve d'une bonne dose d'équilibrisme: recruter des salariés motivés, avec des compétences linguistiques, relationnelles, informatiques, "présentant bien», tout en acceptant d'être payés au Smic et de travailler dans un contexte organisationnel très difficile et sans avoir de véritables perspectives de carrière dans l'entreprise.

\section{Serviabilité et déférence pour sauver les apparences}

L'écart entre le service réel - lié à l'organisation $\mathrm{du}$ travail et aux trajectoires professionnelles des salariés - et le service affiché - qui découle de la politique commerciale de l'hôtel - façonne le travail des salariés du front office. Tout d'abord, ceux qui ont des postes à responsabilité ou qui aspirent à en occuper sont censés être très flexibles et polyvalents car les départs du personnel pour démission, licenciement ou non confirmation à la fin de la période d'essai sont fréquents. De ce fait, non seulement les responsables forment constamment de nouveaux embauchés mais eux-mêmes sont aussi amenés à effectuer des heures supplémentaires. Comme nous l'avons déjà indiqué, le personnel de la réception est en règle générale très polyvalent: les femmes, qu'elles soient réceptionnistes, chefs de réception ou assistantes de direction, donnent un coup de main au restaurant pour le petitdéjeuner ou montent aux étages pour aider une femme de chambre à terminer la préparation des chambres. Elles peuvent même monter les bagages des clients lorsque le bagagiste est trop occupé.

Par ailleurs, nous avons observé que les salariés sont particulièrement déférents envers les clients, ne serait-ce que parce qu'ils sont conscients que les services offerts ne sont pas toujours de bonne qualité. Ils répètent souvent: "Ici, on fait du cinéma!» Alors que le personnel du front office rencontre des difficultés à la réception, liées par exemple à l'attribution des chambres ou aux pannes techniques, nous avons remarqué comment, tout à fait spontanément, certains salariés vont vers le client en affichant un large sourire et la volonté de se mettre à leur service. En effet, les salariés doivent non seulement masquer leurs sentiments négatifs engendrés par la fatigue et le stress, mais aussi afficher un enthousiasme sans faille, censé témoigner de leur plaisir d'être en interaction avec les clients (HochschILD, 1983).

Ce travail physique et émotionnel s'inscrit à l'intérieur d'un système d'interactions très asymétriques. Les salariés essaient de satisfaire les clients pour ne pas avoir à gérer leur mécontentement mais aussi pour garder leur emploi. En effet, ce qui différencie l'hôtellerie de luxe de l'hôtellerie de niveau inférieur, est le fait que le client insatisfait puisse s'en prendre de manière très brutale aux salariés du front office. Nous avons été confrontés, à plusieurs reprises, à la violence verbale des clients. Ces situations désagréables peuvent certes se produire dans n'importe quelle entreprise de services mais, dans l'hôtellerie de luxe, le management peut légitimer de tels comportements; ajoutons également qu'il arrive que des clients écrivent des lettres pour demander le 
licenciement d'un salarié qu'ils ont trouvé impoli ou trop peu professionnel. Dans une relation de service très asymétrique, comme c'est le cas ici, les défaillances organisationnelles et les erreurs des salariés inexpérimentés augmentent les risques d'avoir affaire à la violence verbale des clients (voir encadré 5). Qu'ils soient ou non responsables du mécontentement des clients, les salariés sont censés non seulement ne pas protester, mais aussi présenter des excuses.

\section{Encadré 5 Caprice de client}

Chargé d'amener un room service dans une chambre, nous avons récupéré le plateau, préparé par un équipier, au monte-charge avant de l'apporter aux clients, un couple plutôt âgé. Alors que nous étions sur le point de sortir, le monsieur nous demanda, en anglais, si en France, nous ne mangions pas de pain, ce qui était sa manière de nous faire remarquer que la corbeille de pain avait été oubliée. Nous nous sommes donc précipité vers la cuisine, au rez-de-chaussée, pour la récupérer. De nouveau dans la chambre, et de nouveau sur le point de sortir, le monsieur nous fit cette fois remarquer que le sel n'était pas non plus sur le plateau... Bref, l'un dans l'autre, le client nous a contraint à quatre allers-retours successifs afin d'apporter les éléments qui manquaient à sa commande au lieu d'en dresser la liste complète dès le début. D'après nos observations, ce genre de comportements, quelque peu pervers, est très fréquent. Le salarié est censé maintenir son sang-froid s'il veut conserver son poste de travail.

Lorsque le client est mécontent, le salarié se doit également de montrer de l'empathie en usant de formules telles que «si j'étais à votre place, je serais bien déçu de ce service», "je comprends tout à fait vos raisons ", etc. L'insatisfaction du client, souvent générée par l'organisation du travail même au sein de l'hôtel, peut donc se traduire par des situations difficiles à gérer pour le salarié. Que ce soit en raison de problèmes techniques en chambre, d'une faible maitrise de la langue anglaise ou du logiciel de gestion hôtelière de la part d'un salarié «non professionnel», de la «scénographie ${ }^{(26)}$ 》 de l'hôtel ou de la pression des clients à la réception, le professionnalisme du salarié a de nombreuses occasions d'être remis en question de façon peu amène, comme le montre l'épisode relaté ci-après qui a eu lieu un dimanche (voir encadré 6) :

Obligés d'endosser des responsabilités qui ne devraient pas leur incomber, et, par conséquent, de supporter les plaintes des clients et les réprimandes de la hiérarchie, les salariés des services

(26) L'aménagement et la décoration de l'hôtel rendent parfois plus pénibles les conditions de travail du personnel. La réception «design», minimaliste, est peu spacieuse : les salariés du front office n'ont donc objectivement pas la place suffisante pour ranger les documents dont ils ont besoin et sont contraints de passer la plupart du temps debout. doivent se livrer à un important «travail émotionnel» (Hochschild, 1983). Il ne s'agit certes pas d'une particularité du secteur hôtelier de luxe. Néanmoins, le fait que les humiliations publiques

\section{Encadré 6}

\section{Quand les obstacles s'accumulent...}

Un après-midi, alors que nous étions en train de nous occuper de la réservation de plusieurs taxis, nous reçûmes un appel téléphonique d'Angleterre d'un monsieur qui nous demanda de le mettre en communication avec un client. Or, impossible de retrouver le nom de celui-ci dans le logiciel de gestion hôtelière (1) après qu'il eut changé de chambre suite à une fuite d'eau : la personne en charge de l'affaire avait oublié de mettre à jour le logiciel. En outre, le monsieur au téléphone ne connaissait pas non plus le numéro de la nouvelle chambre car la personne qu'il cherchait à joindre n'avait pas su le lui communiquer; la décoration de cet hôtel a été prévue de telle façon que le numéro des chambres n'est pas indiqué en gros sur la porte mais en tout petit en bas si bien que les clients ne le voient pas forcément. En fait, en lieu et place d'un numéro classique, c'est le nom d'un personnage de l'histoire française qui est inscrit. Notre correspondant essaya de nous dire ce nom mais sa prononciation était tellement mauvaise qu'il en était incompréhensible (d'ailleurs, personne parmi les membres de la réception ne parvenait à faire l'association entre ces noms et les numéros de chambre - on se servait toujours des numéros pour identifier les chambres). N'oublions pas que, dans le même temps, nous avions à gérer les clients qui attendaient avec impatience l'arrivée d'un taxi. Notre interlocuteur, que nous ne mettions toujours pas en relation avec son correspondant à Paris, rappela plusieurs fois tant et si bien que ce dernier, un jeune homme d'une vingtaine d'années venu passer le week-end à Paris avec sa petite amie, sans doute prévenu par SMS qu'on cherchait à le joindre, finit par descendre dans le hall, en peignoir et pieds nus, pour prendre l'appel à la réception, au vu et au su de tout le monde, non sans avoir clamé haut et fort que nous ne savions pas faire notre travail. Reçu le lendemain à sa demande par le manager pour avoir des explications, il se plaignit vivement de notre conduite, d'autant qu'il s'agissait là, d'après lui, d'un important «coup de fil professionnel ».

(1) Le logiciel de gestion hôtelière a trois fonctions essentielles. La première dresse un panorama statique de l'hôtel, qui renseigne notamment sur l'identité des clients présents dans chaque chambre. Les chambres en arrivée ou en départ y sont mises en évidence par des couleurs différentes. Dès que le dernier client est arrivé ou, au plus tard, à 2 heures du matin, le night audit réalise la clôture informatique de la journée hôtelière qui sera donc archivée. La deuxième fonction permet de gérer les réservations. La dernière fonction est utilisée principalement par la gouvernante, qui informe ainsi la réception sur l'état des chambres (sale, en recouche, hors service, en travaux, etc.). Nous avons toutefois remarqué qu'assez régulièrement, la gouvernante tient les réceptionnistes au courant au fil de l'eau via talkie-walkie avant même d'actualiser les données entrées dans le logiciel. 
soient acceptées caractérise ces établissements haut de gamme. L'accusation en public des salariés, dont le professionnalisme est remis en question par les clients, contribue à façonner les relations à l'intérieur de l'équipe et avec les clients. Même si sa survenue reste occasionnelle, combinée à la distance sociale séparant clients et salariés, elle contribue à fragiliser l'identité professionnelle des derniers.

$\mathrm{Au}$ quotidien, une bonne partie du travail relationnel des salariés en interaction avec les clients se traduit par des pratiques de déférence, sortes de rituels visant à sacraliser le «moi» du client (GoFFMAN, 1974). L'organisation du travail d'accueil de l'Hôtel Fashion reproduit en partie le modèle de l'accueil dans les palaces parisiens, qui lui-même réplique, de la manière la plus accomplie qui soit, l'organisation du travail domestique des maisons de l'aristocratie et de la haute bourgeoisie (Élias, 1985 ; Veblen, 1970). Au-delà de la beauté de l'immeuble et de sa situation en centreville, ce qui fait le luxe, du moins sous l'angle du service, ce n'est pas seulement la personnalisation des prestations, mais une certaine ritualisation des interactions entre clients et salariés. En premier lieu, les salariés contribuent à mettre en valeur le client grâce à un ensemble de comportements symboliques : ils sont ainsi censés ouvrir la porte $\mathrm{du}$ hall lors du passage des clients, les accompagner jusqu'au taxi et leur ouvrir la portière, porter leurs bagages, garer leur voiture, leur céder toujours le passage, descendre de l'ascenseur lorsqu'ils doivent y monter, aller les chercher avec un parapluie ouvert lorsqu'il pleut, s'éloigner de l'hôtel ou se cacher pour prendre leurs pauses cigarettes ou téléphoner. Ils doivent aussi manifester leur déférence par un ensemble d'expressions du visage et d'attitudes corporelles : le sourire, mais aussi d'autres mimiques témoignant disponibilité, patience, attention, comme le fait d'incliner la tête vers les clients pour souligner la position d'écoute ou bien de garder les mains serrées au niveau du bassin (voir encadré 7). Les formules de politesse sont en outre scrupuleusement respectées.

Le fait que les clients soient déchargés du port du moindre objet - en effet, le bagagiste est censé prendre en charge même des petits sacs ou des bagages très légers qui ne fatigueraient nullement les clients - prend une signification hautement symbolique qui dépasse la simple commodité.

\section{Encadré 7}

\section{L'entrée dans l'univers enchanté du luxe}

Les salariés accomplissent également toute une série de tâches afin de marquer l'entrée du client dans l'univers enchanté du luxe. Le check-in est mis en scène de telle sorte que les clients aient pleinement conscience que, désormais, ils n'ont plus à s'occuper ni de leurs bagages ni de leur voiture. Après s'être adressé aux clients avec un sourire, le bagagiste prend en charge leurs bagages et les libère ainsi de tout travail physique; puis, les mains serrées au niveau du bassin, se tenant bien droit auprès des bagages déposés à côté de la réception, il attend que le réceptionniste ou le concierge ait accompagné les clients faire un tour du hall. Le réceptionniste accompagne ensuite les clients en chambre en leur rappelant que leur collègue, dont il cite le prénom, y montera leurs bagages cinq minutes plus tard; le travail physique de portage est donc tenu loin des yeux des clients pendant qu'ils visitent l'hôtel et leur chambre. C'est seulement après leur installation que le bagagiste frappe doucement à leur porte pour amener les bagages et, en l'occurrence, recevoir un pourboire.

En outre, la déférence envers les clients se manifeste à travers le soin apporté à la «présentation de soi »(Goffman, 1973). Enjeu de tensions importantes au sein de l'établissement, tout écart par rapport aux normes fait l'objet de sanctions et peut conduire au licenciement du salarié ou de la salariée (27). Ainsi, le personnel doit bien repasser ses chemises; les hommes doivent avoir la barbe soigneusement rasée avant chaque service, les cheveux courts ou en tout cas bien coiffés et les chaussures cirées de manière irréprochable. Notons que l'hôtel ne fournissant pas d'uniforme, les salariés doivent acheter leurs propres chemises, chaussures et costume, ce qui représente un investissement important vu qu'ils n'ont aucune certitude d'être embauchés à la fin de la période d'essai. Enfin, les femmes doivent éviter les boucles d'oreilles trop visibles, attacher leurs cheveux et travailler presque toute la journée debout avec des talons mesurant au minimum huit centi-

(27) De nombreux salariés ont été renvoyés en raison de problèmes concernant leur présentation: citons le cas d'un employé du bar qui portait des chaussures marron plutôt que noires, d'un night audit qui avait mauvaise mine à cause de la fatigue et du stress - il faisait des études pendant la journée -, d'un autre qui, d'après la directrice, était trop gros et trop vieux il avait la quarantaine-, d'un bagagiste qui, refusant de rester tout le temps debout, s'installait dans les fauteuils du hall en attendant les clients, d'un autre qui avait refusé de se raser la barbe durant la période d'essai ou, enfin, d'un bagagiste qui avait acheté un costume «trop moche». Nous-mêmes avons reçu de nombreuses critiques, de la part des responsables ou même des réceptionnistes, sur nos cheveux jugés trop longs et sur notre barbe sur peau mate. Les candidats de plus de 35 ans avaient généralement du mal à se faire embaucher: l'assistante de direction, à qui il est arrivé de faire le tri des CV de candidatures en notre présence, répétait souvent à propos des candidats d'un certain âge : «Comment ils peuvent se présenter avec cette tête? " 
mètres de haut. Le personnel de l'hôtellerie haut de gamme est donc contraint d'assurer une mise en scène de l'accueil propre à l'univers du luxe tout en travaillant dans l'urgence et sans disposer des mêmes ressources organisationnelles que le personnel des hôtels les plus prestigieux.

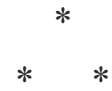

Parmi les nombreux hôtels sur le marché en expansion de l'hôtellerie de luxe, certains offrent un service que nous pouvons caractériser, à la suite de notre étude, de luxe bas de gamme. Ces hôtels revendiquent des prestations hautement soignées et personnalisées, à l'image de celles proposées dans les palaces occupant le sommet du marché, mais le modèle d'emploi en vigueur, ainsi que l'organisation du travail, ne permettent pas toujours de les mettre en œuvre. Le modèle organisationnel y est en effet très différent dans la mesure où autonomie et professionnalisme des salariés y sont faibles et où le management essaie de rationaliser le travail des salariés du front office tout en leur imposant d'être très flexibles et polyvalents. D'ailleurs, la rigidité organisationnelle et la gestion expéditive de la main-d'œuvre ne semblent pas favoriser un engagement de fond de l'ensemble des salariés dont la plupart souhaitent quitter leur emploi dès qu'ils auront trouvé mieux ailleurs. Les tensions entre les employés des différents métiers de l'hôtel occasionnent des oublis et des manques dans le service. Nous avons ainsi montré que le modèle d'emploi et de travail en vigueur dans ces hôtels, dits " haut de gamme» mais délivrant un service de luxe au rabais, emprunte davantage de caractéristiques à l'hôtellerie de chaîne et à la restauration rapide qu'à l'hôtellerie de grand luxe: faible expérience professionnelle, précarité de l'emploi notamment pour les employées de back office (femmes de chambre), polyvalence poussée à l'extrême de tous les salariés. En revanche, on retrouve bien la «division sexuée et ethnique du travail» commune à l'ensemble du secteur de l'hôtellerie-restauration. Les salariés non professionnels du front office se différencient néanmoins du personnel volatile d'autres entreprises de services du fait qu'ils disposent d'un capital culturel important. Leurs compétences linguistiques et relationnelles permettent à l'hôtel de se distinguer par rapport à l'hôtellerie de chaîne aux yeux des clients étrangers, de plus en plus nombreux, qui attendent des prestations conformes aux critères en vigueur dans les hôtels de luxe.

Au-delà de la beauté des lieux, ce qui permet de sauver les apparences et de garantir l'enchantement de l'expérience des clients est l'implication de façade des salariés. Leur déférence et leur serviabilité permettent de respecter les codes formels du service de luxe en sacralisant le «moi» des clients. Nos analyses ne prétendent pas représenter l'ensemble du secteur de l'hôtellerie haut de gamme : parmi les hôtels que nous avons étudiés, également par observation participante, l'Hôtel du Prince se rapproche en effet davantage du modèle "hiérarchique professionnel» de l'hôtellerie de grand luxe (SHERMAn, 2007), dans lequel les salariés non professionnels n'ont que rarement accès aux postes de travail du front office. Si le marché du tourisme de luxe est très segmenté, et malgré l'introduction d'un nouveau classement hôtelier, l'organisation du travail et le niveau de prestations varient beaucoup selon les hôtels au sein d'une même catégorie. Quoi qu'il en soit, notre enquête souligne que l'expansion du secteur haut de gamme est loin d'être toujours synonyme d'une amélioration des conditions d'emploi et de travail, même sur les postes de front office.

\section{Bibliographie}

Adler P. A., Adler P. (2004), Paradise laborers : hotel work in the global economy, Ithaca, ILR Press.

Eckert E., Monchatre S. (2007), «Mixité, polyvalence et formes sexuées de la division du travail de l'hôtellerierestauration de chaîne à la chaîne de montage automobile », in Aballea F., Lallement M. (coord.), Relations au travail, relations de travail, Toulouse, Octarès, pp. 229-236.

Élias N. (1985), La société de cour, Paris, Flammarion.

Ferreira De Macêdo M. B. (2003), «Femmes de ménage et veilleurs de nuit: une approche sexuée du travail précaire dans un hôtel en France», Cahiers du genre, n 35 , pp. 189-208.

Ferreira De Macêdo M. B., Lada E., Kergoat D. (2007), La précarisation de la santé au travail et trajectoires professionnelles: le cas des femmes de chambre et des veilleurs de nuit, Rapport, Paris, Ministère de la Santé et des Sports.

ForTino S. (1999), «De la ségrégation sexuelle des postes à la mixité au travail : étude d'un processus », Sociologie du travail, vol. 41, $\mathrm{n}^{\circ} 4$, pp. 363-384.

GADREY J. (1994), «La modernisation des services professionnels. Rationalisation industrielle ou rationalisation professionnelle?», Revue française de sociologie, vol. 35, $\mathrm{n}^{\circ}$ 2, pp. 163-195.

GADREY J. (coord.) (2002), Hôtellerie-restauration: héberger et restaurer l'emploi. Les cas français, américain et japonais, Paris, La Documentation française. 
GiRAud C. (2007), «Recevoir le touriste en ami : la mise en scène de l'accueil marchand en chambre d'hôte», Actes de la recherche en sciences sociales, $\mathrm{n}^{\circ} 170$, pp. 14-31.

Goffman E. (1973), La mise en scène de la vie quotidienne. Tome I: La présentation de soi, Paris, Éditions de Minuit.

Gofmann E. (1974), Les rites d'interaction, Paris, Éditions de Minuit.

Guegnard C., Meriot S.-A. (2009), «Alice au pays des hôtels : de l'autre côté du miroir», in Caroli E., Gautié J. (dir.), Bas salaires et qualité de l'emploi : l'exception française?, Paris, Éditions Rue d'Ulm, pp. 269-332.

Hochschild A. R. (1983), The managed heart: the commercialization of human feeling, Berkeley, University of California Press.

Hughes E. C. (1996), Le regard sociologique: essais choisis / Everett C. Hughes; textes rassemblés et présentés par Jean-Michel Chapoulie, Paris, École des hautes études en sciences sociales.

Kotsi F. (2007), «Les souvenirs religieux du mont Athos. La frontière entre symboles sacrés et objet économiques », Actes de la recherche en sciences sociales, $\mathrm{n}^{\circ} 170$, pp. $48-57$.

LADA E. (2009), «Divisions du travail et précarisation de la santé dans le secteur hôtelier en France : de l'action des rapports sociaux de sexe et autres rapports de pouvoir », Travailler, $\mathrm{n}^{\circ} 22$, pp. 9-26.

Lipovetsky G., Roux E. (2003), Le luxe éternel. De l'âge du sacré au temps des marques, Paris, Gallimard.

Molinari-Perrier M. (2010), «L'insertion des jeunes dans l'hôtellerie-restauration», Net.Doc, $\mathrm{n}^{\circ} 73$.

Monchatre S. (2006), «Instrumentalisation des femmes au travail et de travail par les femmes de l'hôtellerierestauration», in Flahault É. (dir.), Couppié T. (collab.), L'insertion professionnelle des femmes : entre contraintes et stratégies d'adaptation, Rennes, Presses universitaires de Rennes, pp. 231-242.

Monchatre S. (2010), Etes-vous qualifié pour servir?, Paris, La Dispute.

ODIT (2007), Le marché du tourisme de luxe : évolution des clientèles et de leurs attentes. Impacts, menaces et opportunités pour le tourisme français, Paris, Observation, développement et ingénieries touristiques (ODIT) France.

Peretz H. (1992), «Le vendeur, la vendeuse et leur cliente. Ethnographie du prêt-à-porter de luxe», Revue française de sociologie, vol. 33, $\mathrm{n}^{\circ}$ 1, pp. 49-72.

Peretz H. (2005), «Soldes "haut de gamme" à Paris», Ethnologie française, vol. 35, $\mathrm{n}^{\circ} 1$, pp. 47-54.

Poupeau F., RÉAu B. (2007), «L'enchantement du monde touristique», Actes de la recherche en sciences sociales, $\mathrm{n}^{\circ} 170, \mathrm{pp} .4-13$.

Puech I. (2004), «Le temps du remue-ménage. Conditions d'emploi et de travail des femmes de chambres», Sociologie du travail, vol. 46, $\mathrm{n}^{\circ} 2$, pp. 150-167.

RÉAU B. (2006), «Relations amicales et rapports marchands: la relation de service dans les clubs de vacances », in Durand J.-P., Le Floch M.-C. (dir.), La question du consentement au travail. De la servitude volontaire à l'implication contrainte, Paris, L'Harmattan, pp. 131-140.

RÉAu B. (2007), «S'inventer un autre monde : le Club Méditerranée et la genèse des clubs de vacances en France (1930-1950)», Actes de la recherche en sciences sociales, $\mathrm{n}^{\circ} 170, \mathrm{pp}$. 66-88.

RÉAu B. (2011), Les Français et les vacances. Sociologie des pratiques et offres de loisirs, Paris, CNRS éditions.

SHERMAN R. (2007), Class acts. Service and inequality in luxury hotels, Berkeley, University of California Press.

Veblen T. (1970), Théorie de la classe de loisir, Paris, Gallimard. 